\title{
LOW PERFORMER
}

\section{Minderleitung führt zu Minderleistung}

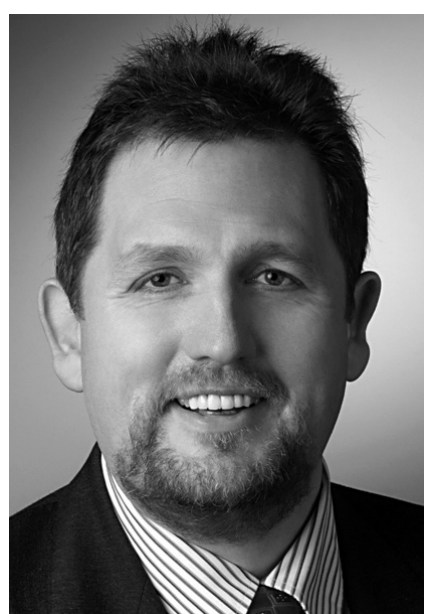

VON FRIEDHELM WENSING Friedhelm Wensing ist Kaufmännischer Vorstand des Eylarduswerkes, einer diakonischen Einrichtung der Kinder-, Jugendund Familienhilfe in Bad Bentheim mit ca. 340 Mitarbeitenden und knapp 700 betreuten Personen. www.eylarduswerk.de

\author{
Mitarbeitende, die nicht die geforderte Leistung \\ erbringen wollen oder können, stellen für eine \\ Führungskraft eine besondere Herausforderung dar. \\ Der schlechteste Rat dabei ist wegzusehen, denn dies \\ belastet die Nutzer sozialer Dienste und Einrichtungen, \\ die beteiligten Kolleginnen und Kollegen sowie \\ die Organisation als Ganzes. Gefragt sind dagegen \\ Einfühlungsvermögen und konsequentes Handeln.
}

Eine Gallup-Studie aus dem Jahr 2010 belegt, dass nur elf Prozent der deutschen Beschäftigten engagiert arbeiten. Ganze 66 Prozent spüren ihrem Arbeitgeber gegenüber keine Verpflichtung und machen Dienst nach Vorschrift. Die Gründe hierfür sind hausgemacht: Es fehlt an Förderung, Feedback und Lob.

Der Begriff »Low Performer « ist weder aus sich heraus noch durch seine Übersetzung geeignet, Mitarbeitende für jedermann nachvollziehbar zu beschreiben und einzuordnen. Die in der Praxis im Zusammenhang mit einem Low Performer am häufigsten auftretenden Erscheinungsformen sind Schlechtund Minderleistung. Liegt eine dieser Erscheinungsformen vor, wird das Verhältnis von Leistung und Gegenleistung im Austauschverhältnis zwischen Arbeitgeber und Arbeitnehmer gestört. So zeigen sich im täglichen Arbeitsablauf Störungen, die häufig erst dann erkannt werden, wenn sie zu mehr oder minder schweren Schäden führen. Den volkswirtschaftlichen Schaden durch die Folgen innerer Kündigung schätzen die Autoren der Gallup-Studie auf bis zu 124 Milliarden Euro.

Allerdings bedenken nur wenige Führungskräfte, dass diese Mitarbeiterinnen und Mitarbeiter einmal zur Einrichtung gepasst haben. Sie wurden von ihnen eingestellt und haben ihre Probezeit erfolgreich absolviert. Warum bringen sie plötzlich nur noch Mindestleistung und trifft bei dieser Entwicklung wirklich nur die Low Performer die Schuld? Nicht selten erweist sich die schlechte Leistung als Folge mangelnder Führung (»Minderleitung «).

Leitungs- und Führungskräfte sehen sich oftmals Situationen ausgesetzt, die den Anschein erwecken, als gelänge ihnen kaum mehr der Zugang zu offenbar leistungsschwachen Mitarbeiterinnen und Mitarbeitern. Häufig werden sie vom Team »mitgeschleppt «, weil Vorgesetzte nicht wissen, wie sie mit sogenannten Low Performern umgehen sollen. Sie tolerieren leistungsschwache Mitarbeitende auch, weil sie eine Konfrontation scheuen oder nicht wissen, was sie tun sollen. Wenn Low Performer »mitgeschleppt « werden, wird das von vielen Mitarbeiterinnen und Mitarbeitern als unfair empfunden, weil sie beispielsweise Kosten durch Arbeitsausfall und Fehlleistung verursachen. Sie sind Negativ-Beispiele für ihre Kolleginnen und Kollegen mit unmittelbarer Wirkung für die Organisation, das Team und die Führungskraft selbst.

Eine Auseinandersetzung mit Gutleistung und Schlechtleistung gibt es in vielen sozialen Organisationen kaum. Leistung sollte jedoch immer überprüfbar sein. Viele Mitarbeitende kennen nicht die Leistungsanforderungen, die an sie gestellt werden. Stellen- und Aufgaben- 
beschreibungen kennzeichnen vielfach nicht die zu erbringende Leistung im Sinne von Arbeitsergebnis.

Mitarbeiterinnen und Mitarbeiter sind grundsätzlich bestrebt, dass ihre Leistungen geschätzt und anerkannt werden. Sie sind dem Grunde nach deshalb auch leistungsbereit. Es gibt viele Ursachen und Einflüsse, die die Leistungsfähigkeit von Mitarbeitenden tangieren und aus ehemals leistungsstarken Kolleginnen und Kollegen Low Performer werden lassen können. Diese können sowohl aus den persönlichen Bereichen der Mitarbeitenden als auch aus dem Gebiet des Arbeitgebers kommen. Auf Umstände, die aus dem familiären oder persönlichen Umfeld stammen, hat der Arbeitgeber geringere Einflussmöglichkeiten als auf die Umstände, die aus seiner Sphäre - dem Arbeitsumfeld stammen.

Die Art der Identifikation von Mitarbeitenden mit der Organisation beeinflusst ihre Leistungsbereitschaft sehr stark - im Guten wie im Schlechten. Deshalb gilt es, die emotionale Bindung zur Organisation herzustellen und zu festigen. Ebenso begünstigen mangelnde Unternehmensstrukturen oft »Minderleistungen«. Schlechte Führung und schlechte Kommunikation produzieren reihenweise verbitterte Mitarbeiterinnen und Mitarbeiter, die scheinbar überproportional in sozialen Organisationen anzutreffen sind.

Führungskräfte haben eine ethische Verantwortung "Schlechtleister», die nicht arbeiten wollen, mit Blick auf ihre Kolleginnen und Kollegen konsequent und fair zu behandeln. Häufig besteht in sozialen Einrichtungen eine Verunsicherung im Umgang mit Mitarbeiterinnen und Mitarbeitern, die in ihrer Arbeitsleistung Schwächen aufweisen. Der Umgang mit ihnen ist vielfach tabubehaftet.

Es gibt keinen Königsweg, keinen Automatismus, der aus einem Low Performer einen leistungsstarken Arbeitnehmer macht. Oft genügt es, im Umgang mit dem Low Performer nichts anderes walten zu lassen als den gesunden Menschenverstand. Ihm zu folgen und $\mathrm{zu}$ vertrauen, ist nicht die schlechteste »Führungstechnik«.

Wie die Führungskraft einen Low Performer fördern und motivieren sollte, ist stets von dem konkreten Menschen und der konkreten Situation abhängig. Jeder Low Performer ist ein Individu- um, zu dem ein individueller Zugang gefunden werden muss. Ein positives Menschenbild und die Überzeugung, dass sich jede Mitarbeiterin und jeder Mitarbeiter prinzipiell motiviert und eigeninitiativ für die Interessen seines Unternehmens einsetzt, erleichtern die Führungspraxis.

Um genau zu wissen, ob jemand nur nicht arbeiten kann oder aber nicht will, müssen Führungskräfte sich mit dem Verhalten des Arbeitnehmers, seinen Stärken und Schwächen auseinandersetzen. Wer davon ausgeht, dass jeder Mensch Stärken hat, die gefördert werden können, führt seine Mitarbeitenden zu besseren Leistungen. Das Leitbild für die Kommunikation mit dem leistungsschwachen Mitarbeitenden ist hierbei die »wertschätzende Gesprächsführung «. Auch die Stärken der Low Performer müssen gestärkt, deren Schwächen geschwächt werden.

Wenn Führungskräfte leistungsschwache Mitarbeiter integrieren und ihre Stärken fördern, wirkt sich das nicht nur positiv auf die Organisation oder das Team aus, davon profitieren auch die Mitarbeitenden selber. Durch einen bewussten Umgang mit Low Performern muss es also möglich sein, deren Leistungspotenziale zu fördern. Durch die Kündigung von Low Performer kann eine Angstkultur im Unternehmen entstehen. Wenn Mitarbeiterinnen und Mitarbeiter befürchten müssen, selbst als leistungsschwach identifiziert zu werden, könnten sie demotiviert arbeiten und sich selbst zu Low Performern entwickeln. So lässt sich vorbeugen:

\section{Erster Tipp: Personal gründlich auswählen}

Halten Sie die wichtigsten Anforderungen und Persönlichkeitsmerkmale schriftlich fest. Überprüfen Sie diese gründlich und schließen Sie daran Assessments und strukturierte Interviews an. Begreifen Sie die Probezeit als wirkliche Phase der Erprobung.

\section{Zweiter Tipp: Ziele definieren und kontrollieren}

Verfolgen Sie laufend die Fortschritte Ihrer Mitarbeitenden und machen Sie Leistungen transparent. Dann können Sie im Zweifel schneller reagieren, wenn einmal etwas schief läuft. Mängel sollten sofort angesprochen werden. Beschreiben Sie Lösungswege, zeigen Sie aber auch jederzeit unmissverständlich Fehler auf. Loben Sie unbedingt auch kleinere Fortschritte. Sprechen Sie notfalls Konsequenzen an, falls die gewünschten Leistungen nicht eintreten.

\section{Dritter Tipp: Zeit lassen}

Geben Sie dem Low Performer genügend Zeit, die angesprochenen Maßnahmen umzusetzen. Niemand wird mit einem Aufwand von täglich fünf Minuten zur Spitzenkraft. Kalkulieren Sie dabei auch Rückschläge ein. Es ist noch kein Meister vom Himmel gefallen.

\section{Vierter Tipp: Konsequenzen einleiten}

Wenn alle Anstrengungen nicht zu den gewünschten Ergebnissen führen, sollten Sie sich von dem Low Performer trennen. Hierbei ist Fairness das oberste Gebot.

\section{Resümee}

Bei allen Schritten ist die Dokumentation unerlässlich. Sie ist die Grundlage für jede weitere Maßnahme. Und sollten diese nicht fruchten, ist sie die Basis, um im Notfall auch rechtliche Schritte zu ergreifen. Wer Fehler oder Mangelleistung in Mitarbeitergesprächen nicht anspricht und in der Personalakte keinerlei Eintrag zu Schlechtleistungen zu finden ist, steht mit leeren Händen da und hat keinerlei Grundlage für eine gegebenenfalls erforderliche Kündigung als letzten Ausweg. Doch sollte sich der Arbeitgeber bewusst sein, dass die Anforderungen hierfür in der deutschen Rechtsprechung sehr hoch sind.

Die Bundesakademie für Kirche und Diakonie bietet mit dem Autor dieses Beitrags eine Veranstaltung über "Low Performer" und dem fairen Umgang mit Minderleistern insbesondere in der Kinder-und Jugendhilfe an. Das Seminar richtet sich an Mitarbeitende mit Leitungsfunktionen und in Geschäftsfübrungen. Es findet statt vom 11. bis 13. November 2013 in Berlin. www.bundesakademie-kd.de 